\title{
Niclosamide induces growth inhibition and apoptosis through down-regulation of PDGFR $\beta$ and STAT3 in human chondrosarcoma cells
}

\section{Xueping Zhang}

the First Affiliated Hospital of Zhengzhou University

Jing Yan

the First Affiliated Hospital of Zhengzhou University

Xiaoning Shao

the First Affiliated Hospital of Zhengzhou University

Yingyu Che

the First Affiliated Hospital of Zhengzhou University

Jingliang Cheng

the First Affiliated Hospital of Zhengzhou University

Xinchang Lu (D luc999@163.com)

the First Affiliated Hospital of Zhengzhou University

\section{Research Article}

Keywords: chondrosarcoma, niclosamide, PDGFRß, STAT3, apoptosis

Posted Date: February 17th, 2022

DOI: https://doi.org/10.21203/rs.3.rs-1354415/v1

License: (1) This work is licensed under a Creative Commons Attribution 4.0 International License.

Read Full License 


\section{Abstract}

Background and objectives Chondrosarcoma is a destructive neoplasm of chondrocytes. Surgery is the principal procedure for this condition. The efficacy of traditional chemotherapy drugs is controversial. Niclosamide is an antihelminthic drug used to treat parasitic infections for nearly 50 years. However recent studies suggested that niclosamide might have the inhibitory effects of cancer.

Methods We investigated the efficaciousness of niclosamide on a chondrosarcoma cell line SW1353.To determine the efficacy of niclosamide on chondrosarcoma, MTT, Flow cytometry, and DAPI assays were used to determine the effects of niclosamide. We determine the activity of niclosamide on the expression of the Raf/MEK/ERK pathway, and the phosphorylation of PDGFR $\beta$ and STAT3.

Results Niclosamide dramatically inhibited cell growth and triggered apoptosis. And niclosamide downregulated the expression of $\mathrm{p}$ - PDGFR $\beta$ and $\mathrm{p}$-STAT3. Furthermore, niclosamide significantly reduced cyclin $\mathrm{D} 1, \mathrm{Rb}$ expressing, which were concurrent with the cell cycle block. We also found a decrease in $\mathrm{p}$ MEK, p-ERK, Bcl-xl, and Bcl-2.

Conclusion These results suggested that niclosamide effectively induced growth inhibition in vitro. And niclosamide could a new therapeutic method for chondrosarcoma.

\section{Introduction}

Chondrosarcoma, always resistant to chemotherapy and conventional chemotherapy, is the second primary neoplasm of bone, but the only primary bone cancer missing effective treatment strategy[1]. The majority of cases more likely occur after age 50 and there was a slight male predominance[2]. The pelvis, particularly the ilium, is more susceptible to chondrosarcoma and only about one percent of chondrosarcomas patients affect the hands and feet. Although limb salvage is preferred, wide surgical resection is still the treatment of choice in the pelvis[3]. About $6 \%$ of the chondrosarcoma patients have distant metastasis when the diagnosis is confirmed and about $25 \%$ of the patient detected with local relapse[4].

Niclosamide was originally developed as a molluscicide. Shortly, scientists found it could effective against human tapeworm infection by inhibiting oxidative phosphorylation and stimulating adenosine triphosphatase activity[5]. US FDA had approved niclosamide in the treatment of worm infections in 1982. Although niclosamide has been used safely for millions of patients, its mechanism does not well elucidated. Accumulated evidence indicated niclosamide has more clinical applications beyond an antihelminthic medication through regulating multiple signaling pathways. However, we know little about the antitumor efficacy of niclosamide on chondrosarcoma.

Platelet-derived growth factor receptor- $\beta$ (PDGFR- $\beta$ ) has a vital role in the tumor microenvironment of many malignancies[6]. PDGFR- $\beta$ is almost up-regulated in fibroblasts in tumor stroma and could serve as a biomarker of the activation status of fibroblasts[7, 8]. PDGFR- $\beta$ signal pathway involved in blood vessel 
formation and early hematopoiesis[9]. The phosphorylated residues of PDGFR- $\beta$ are conducted as the binding sites of the downstream signaling molecules. The phosphorylation of PDGFR- $\beta$ may be related to the depth of cancer invasion[10] and play a major role in the malignant phenotype of chondrosarcoma [5]. The phosphorylation of PDGFR- $\beta$ provides a rationale target for tyrosine kinase inhibitor chemotherapy for tumors [11]. STAT3(Signal Transducer and Activator of Transcription 3) is an important regulator in tumorigenesis, which is frequently elevated in a variety of solid tumors and plays substantial roles in cell cycle progression, apoptosis and tumor angiogenesis[12].In this study, we determined the effect of niclosamide on chondrosarcoma cell line SW1353.

\section{Material And Methods}

Cell culture and reagents.

The human chondrosarcoma cell line, SW1353 cell line, was obtained from American Type Culture Collection. The cell was cultured in Dulbecco's Modified Eagle's Medium (Gibco, USA) supplemented with $10 \%$ fetal bovine serum (Gibco, Invitrogen Corp., USA). Niclosamide was supplied by Sigma Chemical Corporation (St. Louis, MO, USA),

Cell viability and clonogenic assays.

SW1353 cells $\left(5 \times 10^{3} /\right.$ well) were incubated in the 96 -well plates. After being treated with niclosamide or DMSO for 24 hours, 3-(4,5-dimethylthiazol-2-yl)-2,5- diphenyltetrazoliumbromide (MTT) solution (5 $\mathrm{mg} / \mathrm{ml}$ ) was added to the culture medium. Viable cells were detected with an MRX II absorbance reader (DYNEX Technologies, Chantilly, Virginia, USA).

To evaluate cell colony-forming ability, SW1353 cells were incubated in 6-well plates for 10 days before being fixed with methanol and stained with Gentian Violet.

\section{Apoptosis assay}

Apoptotic cells were measured with Annexin V/FITC kit (BD Biosciences, USA) according to the instructions. Samples were analyzed by a flow cytometer (Becton Dickinson, USA).

\section{Cell cycle analysis}

SW1353 cells were seeded in 6-well plates at $2 \times 10^{5}$ per well. After being treated with niclosamide for 24 hours, cells were harvested and washed with cold phosphate-buffered saline (PBS). And then cells were stained with PI for 30 min. Samples were analyzed by flow cytometer (Becton Dickinson, USA).

\section{DAPI assay}

Cells apoptosis was identified after staining with 40,6-diamidino-2-pheny-lindole (DAPI). Condensed chromatin as well as nuclear fragmentation indicate apoptotic nuclei.

\section{Western blotting}


Cells were washed and resuspended in lysis buffer (50 mM Tris- $\mathrm{HCl}, 150 \mathrm{mM} \mathrm{NaCl}, 1 \% \mathrm{NP}-40,2 \mathrm{mM}$ EDTA, $p \mathrm{H} \mathrm{7.4,)} \mathrm{containing} \mathrm{protease} \mathrm{inhibitor} \mathrm{(Amresco,} \mathrm{USA).} \mathrm{Protein} \mathrm{(40} \mu \mathrm{g}$ per lane) was separated by electrophoresis and transferred to a PVDF membrane (Millipore, USA). Following primary antibodies were used in this study: PDGFRß (Cell Signaling Technology), phospho-PDGFRß (Thr751, Cell Signaling Technology), STAT3 (Bioworld Technology Inc.) phospho-STAT3(Ser 727, Bioworld Technology Inc.), phospho-ERK (Thr202/Tyr204, Cell Signaling Technology), phospho-MEK (Ser217/221, Cell Signaling Technology), ERK (Cell Signaling Technology), MEK (Cell Signaling Technology), cyclin D1 (Cell Signaling Technology), Rb (BS1310, Bioworld Technology Inc.), Bcl-2 (sc-783, Santa Cruz), Bax (sc-493, Santa Cruz), Bcl-xl (BS1032, Bioworld Technology Inc.)), Mcl-1 (BS1220, Bioworld Technology Inc.)), $\beta$-actin (sc-1616-R, Santa Cruz), and the appropriate peroxidase-conjugated secondary antibodies were then used, followed by detection by enhanced chemiluminescence (Amersham, USA).

\section{Results}

\section{Niclosamide decreases cells viability and colony-forming ability in SW1353 cells}

Cell proliferation was tested by MTT assay. Niclosamide exerted dose- and time-dependent effects on the proliferation of SW1353 cells (Fig. 1A \& 1B). Niclosamide significantly reduced the colony-forming ability of chondrosarcoma cells after being treated for 24 hours (Fig. 1C \& Fig. 1D).

\section{Niclosamide induce cell apoptosis and induces G0/G1 phase arrest in SW1353 cells}

Niclosamide induced an increase in the percentage of apoptotic cells by a dose-dependent pattern (Fig. 2). Niclosamide-treated cells showed significant nuclear fragmentation, including the typical condensed, fragmented nuclear structure and decreased cell size. This result was also confirmed by the Annexin $V$ apoptosis assay (Fig. 3A \& Fig. 3B).

In addition, to determine the effect of niclosamide on cell cycle distribution. SW1353 cells were treated with niclosamide for 2 hours. As shown in Fig. 3C, niclosamide induced an increase in the G0/G1 population. We also detected the effect of niclosamide on Cyclin D1 and Rb expression in chondrosarcoma cells. The expression level of Cyclin D1 and Rb showed a significant dose-dependent reduction(Fig. 4 ).

\section{Niclosamide reduces phosphorylation of PDGFR- $\beta$ and STAT3 in SW1353 cells}

Total and phosphorylated PDGFR- $\beta$ and STAT3 expression were also investigated in SW1353 cells. After being treated with niclosamide for 12 hours, the total levels of PDGFR- $\beta$ and STAT3 were not significantly 
changed, but p-PDGFR- $\beta$ (Thr751) and p-STAT3(Ser 727) were substantially reduced (Fig. 5).

\section{Niclosamide blocks the RAF/MEK/ERK signaling pathway in SW1353 cells}

We further test the mechanism of niclosamide on the MAPK pathway. Niclosamide could inhibit p-MEK and p-ERK in a dose-dependent manner (Fig. 6).

Effect of Niclosamide on the expression of pro-apoptotic protein Bax, anti-apoptotic proteins Bcl-2, Bcl-xl and $\mathrm{Mcl}-1$

As shown in Fig. 7, after being treated with Niclosamide for 12 hours, niclosamide inhibited expression of $\mathrm{Bcl}-\mathrm{xl}$ and $\mathrm{Bcl}-2$, but there were no changes in expression of $\mathrm{Mcl}-1$ or Bax as shown in Fig. 7.

\section{Discussion}

The major therapy of chondrosarcoma is resection since it is resistant to conventional cytotoxic chemotherapy and radiation [2]. But it is difficult to balance the risk of recurrence and surgical morbidity [3]. And what is more, even low-grade chondrosarcoma may have a high local recurrence rate, let alone the high-grade ones [13].

Niclosamide showed inhibitory effects on multiple signaling pathways, such as Wnt/ $\beta$-catenin, and Notch signaling pathways $[16,17,18]$. Niclosamide also exerts a down-regulation effect on p-STAT3, which played an important role in tumor proliferation and migration [[13]. In this study, we examined the efficacy of niclosamide on SW1353 cells. And the results showed that niclosamide was able to dose-dependently induce growth inhibition and apoptosis in the SW1353 cell line through down-regulation of the expression of p-STAT3 and p-PDGFRß.

The activity of STAT3 is elevated in many solid tumors, and it regulates basic biologic processes in tumorigeneses such as cell cycle, apoptosis, and tumor angiogenesis[12]. Our results suggested that niclosamide inhibited the phosphorylation of STAT3 in a concentration-dependent fashion in SW1353 cells. We further detected the cell cycle analysis. And the results revealed that after treatment with niclosamide, SW1353 cells were arrested in the G1 phase in a dose-dependent manner. Niclosamide also down-regulated cyclin D1 and Rb expression. Cyclin D1 proto-oncogene could be an important regulator of the cell cycles[14]. Rb is a tumor suppressor protein that plays important role in the checkpoint of the cell cycle[15]. We also tested the effect of Niclosamide on cell proliferation by MTT assay, which exerted a marked dose- and time-dependent inhibition. Our results show niclosamide could induce cell apoptosis through the concentration-dependent pattern.

Another interesting target is PDGFR- $\beta$. Aberrant or overexpression of PDGFRs could associate with a variety of diseases [16]. Treatment of PDGFR antagonists through PDGFR inhibition or dephosphorylation and a combination of other therapies looks promising[17]. And it has been proved that PDGFR- $\beta$ plays a 
major role in the malignant phenotype of chondrosarcoma[11]. We investigated the PDGFR- $\beta$ of chondrosarcoma after niclosamide treatment. Our results also proved that niclosamide could inhibit PDGFR- $\beta$ phosphorylation.

Niclosamide could inhibit the RAF/MEK/ERK pathway in a variety of human tumors[18]. The RAF/MEK/ERK pathway is involved in a variety of processes in the cancer cell including cell proliferation and apoptosis. In the current study, niclosamide significantly inhibited phosphorylation levels of MEK and ERK.

The apoptosis effect of niclosamide on SW1353 cells can also be studied. In our study, niclosamide could decrease Bcl-xl and Bcl-2 expression, but do not interfere with the expression of Bax and $\mathrm{Mcl}-1$ proteins. These results suggested that $\mathrm{Bcl}-\mathrm{xl}$ and $\mathrm{Bcl}-2$ may play a crucial role in the niclosamidemediated apoptosis.

In summary, niclosamide could be a potential chemotherapeutic agent for chondrosarcoma by inhibiting proliferation and induction apoptosis. The inhibition of STAT3 phosphorylation, the reduction of the phosphorylation of PDGFR- $\beta$, the inhibition of the RAF/MEK/ERK signaling pathway, and the repression of anti-apoptotic proteins levels may result in the anticancer effects of niclosamide in chondrosarcoma cells SW1353. These observations may provide potentially useful information for the treatment of patients with chondrosarcoma.

\section{Declarations}

Funding: The study was funded by the Scientific and technological project in Henan Province 212102310628.

Conflicts of interest: The authors declare no conflict of interest.

Availability of data and material: The authors confirm that the data supporting the findings of this study are available within the article.

Code Availability: Not applicable.

Authors' Contributions: $\mathrm{X}-\mathrm{pZ}, \mathrm{X}-\mathrm{cL}, \mathrm{Y}-\mathrm{yC}$, and J-IC contributed to planning, design, data collection and data analysis. X-pZ, X-cL, Y-yC, JY, X-nS, and J-IC contributed to interpretation of the results, drafting and revising the manuscript and agree to be accountable for all aspects of the work. Y-yC, JY, X-nS, and J-IC supervised the statistical analyses, $\mathrm{X}-\mathrm{pZ}$ and $\mathrm{X}$-cL curated and analyzed the data and wrote the draft manuscript. All authors contributed to the manuscript and approved the final version.

Consent to participate: Not applicable.

Consent for publication: Not applicable 


\section{References}

1. Zając, A.E., et al., Chondrosarcoma-from Molecular Pathology to Novel Therapies. Cancers, 2021. 13(10): p. 2390.

2. van Praag, V.M., et al., Incidence, outcomes and prognostic factors during 25 years of treatment of chondrosarcomas. Surgical oncology, 2018. 27(3): p. 402-408.

3. Houdek, M.T., et al., Advancing patient age is associated with worse outcomes in low-and intermediate-grade primary chondrosarcoma of the pelvis. Journal of surgical oncology, 2020. 121(4): p. 638-644.

4. Aggerholm-Pedersen, N., et al., Chondrosarcoma: the impact of comorbidity-30 years of experience from a population-based database including 199 consecutive chondrosarcoma patients. Orthopedic research and reviews, 2019. 11: p. 109.

5. Li, Y., et al., Multi-targeted therapy of cancer by niclosamide: $A$ new application for an old drug. Cancer letters, 2014. 349(1): p. 8-14.

6. Andrae, J., R. Gallini, and C. Betsholtz, Role of platelet-derived growth factors in physiology and medicine. Genes \& development, 2008. 22(10): p. 1276-1312.

7. Prakash, J., Cancer-associated fibroblasts: perspectives in cancer therapy. Trends in cancer, 2016. 2(6): p. 277-279.

8. Öhlund, D., E. Elyada, and D. Tuveson, Fibroblast heterogeneity in the cancer wound. Journal of Experimental Medicine, 2014. 211(8): p. 1503-1523.

9. Ribatti, D., B. Nico, and E. Crivellato, The role of pericytes in angiogenesis. International Journal of Developmental Biology, 2011. 55(3): p. 261-268.

10. Suzuki, S., et al., Clinicopathological significance of platelet-derived growth factor (PDGF)- $B$ and vascular endothelial growth factor-A expression, PDGF receptor- $\beta$ phosphorylation, and microvessel density in gastric cancer. BMC cancer, 2010. 10(1): p. 1-10.

11. Mathew P, Thall PF, Bucana CD, Oh WK, Morris MJ, Jones DM, et al. Platelet-derived growth factor receptor inhibition and chemotherapy for castration-resistant prostate cancer with bone metastases. Clin Cancer Res 2007;13(19):5816-5824.

12. Arora L, Kumar AP, Arfuso F, Chng WJ, Sethi G. The Role of Signal Transducer and Activator of Transcription 3 (STAT3) and Its Targeted Inhibition in Hematological Malignancies. Cancers (Basel). 2018 Sep 13;10(9):327.

13. Ren X, Duan L, He Q, Zhang Z, Zhou Y, Wu D, et al.. Identification of Niclosamide as a New SmallMolecule Inhibitor of the STAT3 Signaling Pathway.ACS Med Chem Lett 2010;1(9):454-459.

14. Liao, D.J., et al., Perspectives on c-Myc, Cyclin D1, and their interaction in cancer formation, progression, and response to chemotherapy. Crit Rev Oncog, 2007. 13(2): p. 93-158.

15. Giacinti, C. and A. Giordano, RB and cell cycle progression. Oncogene, 2006. 25(38): p. 5220-7.

16. Pietras K, Rubin K, Sjoblom T, Buchdunger E, Sjoquist M, Heldin $\mathrm{CH}$, et al. Inhibition of PDGF receptor signaling in tumor stroma enhances antitumor effect of chemotherapy. Cancer Res 
2002;62(19):5476-5484.

17. Dai Y, Hartandi K, Soni NB, Pease LJ, Reuter DR, Olson AM, et al. Identification of aminopyrazolopyridine ureas as potent VEGFR/PDGFR multitargeted kinase inhibitors. Bioorg Med Chem Lett 2008;18(1):386-390.

18. Lee MC, Chen YK, Hsu YJ, Lin BR. Niclosamide inhibits the cell proliferation and enhances the responsiveness of esophageal cancer cells to chemotherapeutic agents. Oncol Rep. 2020 Feb;43(2):549-561.

\section{Figures}
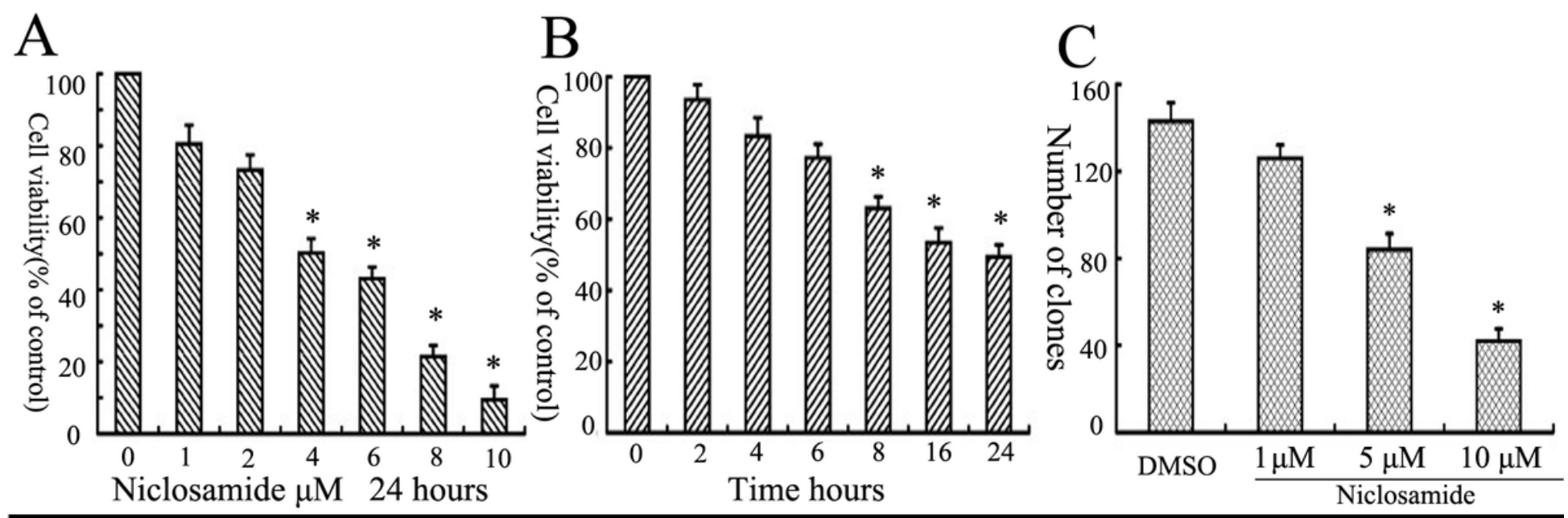

\section{DMSO}

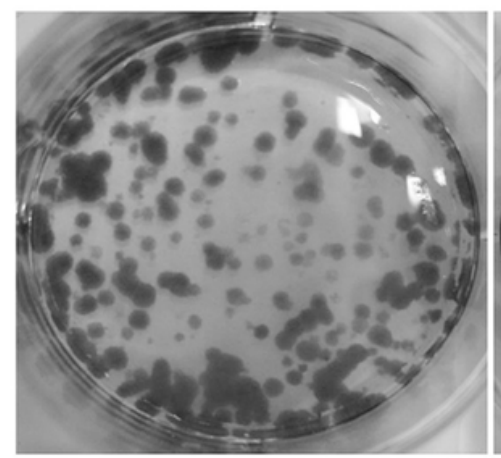

Niclosamide $1 \mu \mathrm{M}$

Niclosamide $5 \mu \mathrm{M}$

Niclosamide $10 \mu \mathrm{M}$
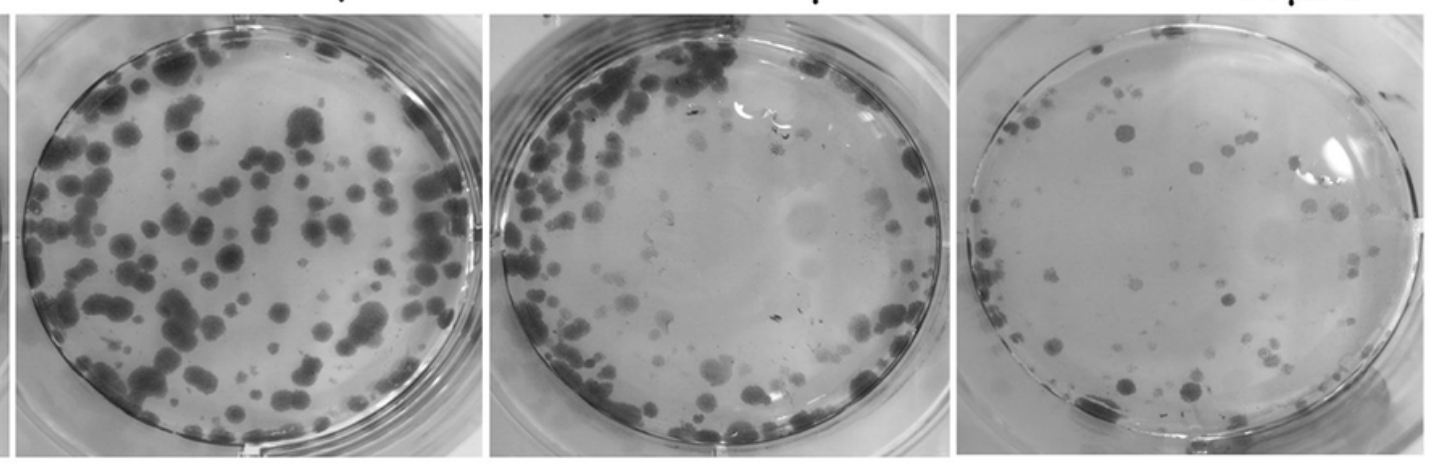

\section{Figure 1}

Effect of Niclosamide on cell viability of human chondrosarcoma cells. (A) SW1353 cells were incubated with $0,1,2,4,6,8$ or10 $\mu \mathrm{M}$ Niclosamide for $24 \mathrm{~h}$. (B) SW1353 cells were incubated with $5 \mu \mathrm{M}$ Niclosamide for $2 \mathrm{~h}, 4 \mathrm{~h}, 8 \mathrm{~h}, 16 \mathrm{~h}$, and $24 \mathrm{~h}$. At the end of incubation, the cell survival rates were determined by MTT methods. Cell viability is expressed as the percentage of cell survival compared with the control. (C)Cell viability was measured by clonogenic assay. Representative images of colony formation and stained by crystal violet, and results displayed in histogram form(D). Data are from three independent experiments. ${ }^{*} p<0.05$, compared to the control group. 

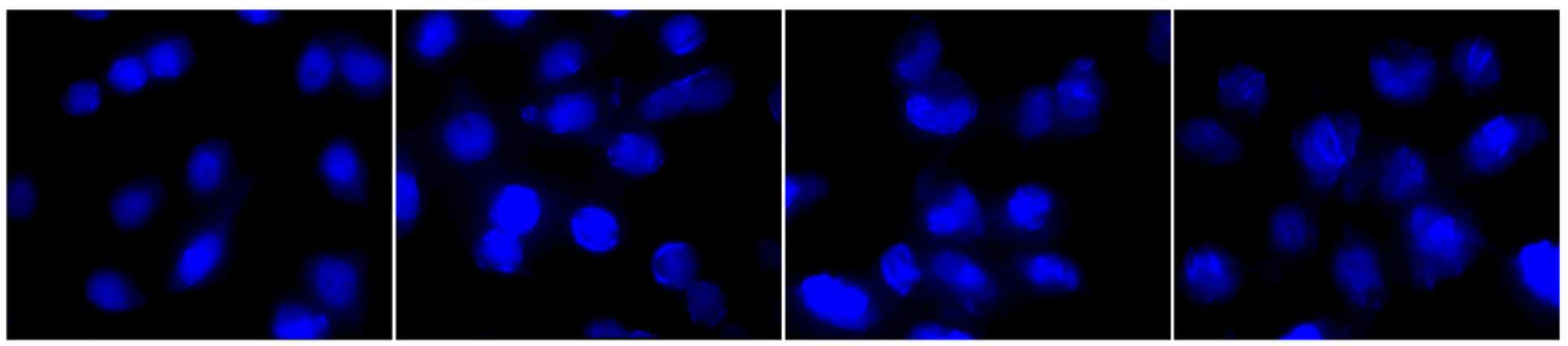

Figure 2

DAPI staining of SW1353 cells after exposure to Niclosamide. The induction of apoptotic cell death was accompanied by characteristic morphological and structural changes, including a condensed and fragmented nuclear structure and decreased cell size. (original magnification $\times 200$ ).
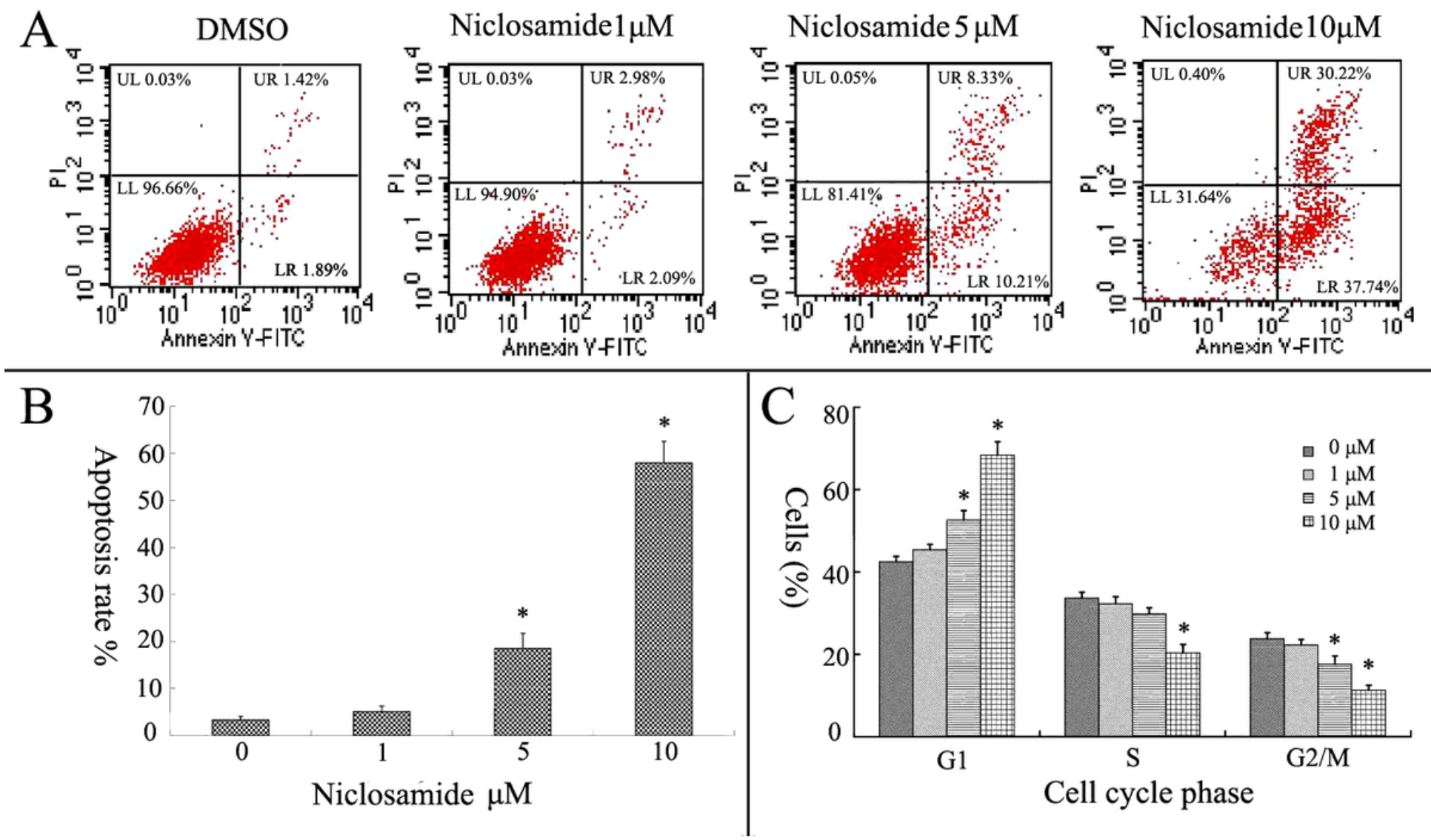

\section{Figure 3}

Niclosamide caused apoptotic death in SW1353 cells. After 24 hours of treatments with Niclosamide, cells were collected and stained with Annexin V/PI followed by FACS analysis. Representative FACS analysis scatter-grams of Annexin V/PI stained $0,1,3$, and $5 \mu \mathrm{M}$ Niclosamide treatment showed four different cell populations marked as double negative (unstained) cells showing live cell population (LL, 
lower left), Annexin $V$ positive and PI negative stained cells showing early apoptosis (LR, lower right), Annexin V/PI double-stained cells showing late apoptosis (UR, upper right), and finally PI-positive and Annexin $V$ negative stained cells showing dead cells (UL, upper left). Results were presented as mean \pm standard as shown in the histogram. ${ }^{*} \mathrm{P}<0.05$ compared with control.

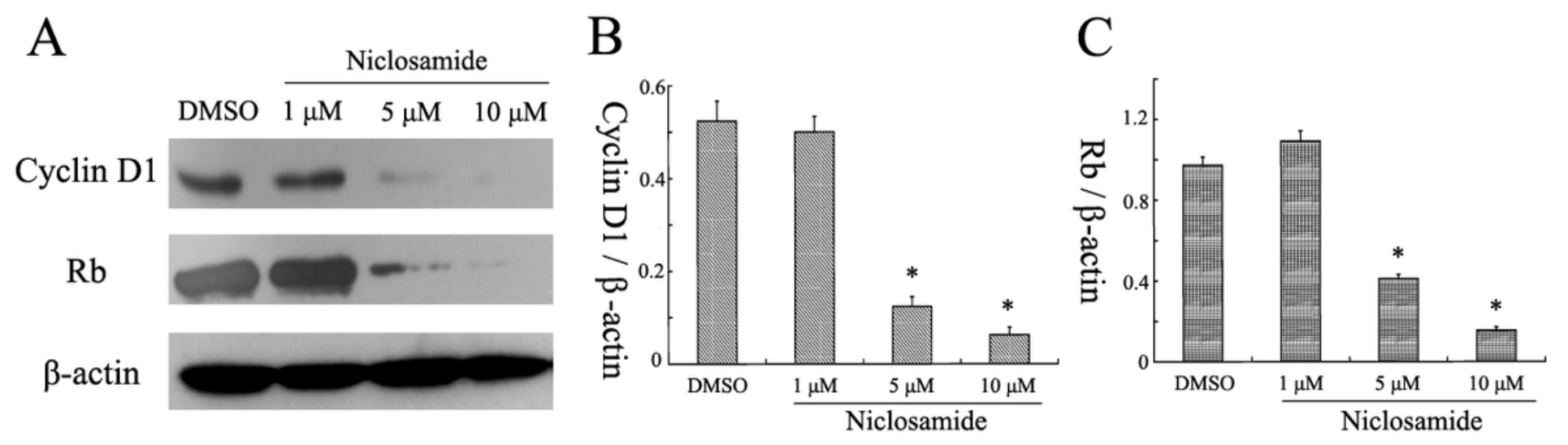

Figure 4

Niclosamide down-regulates Rb and cyclin D1 in SW1353 cells. SW1353 cells were treated with Niclosamide for 12 hours in DMEM containing 10\% FBS. $\beta$-actin expression was used to assess equal lane loading. Densitometric assays of Rb and cyclin D1 were performed and data were presented as mean \pm standard as shown in the histogram. ${ }^{*}<<0.05$ compared with control.
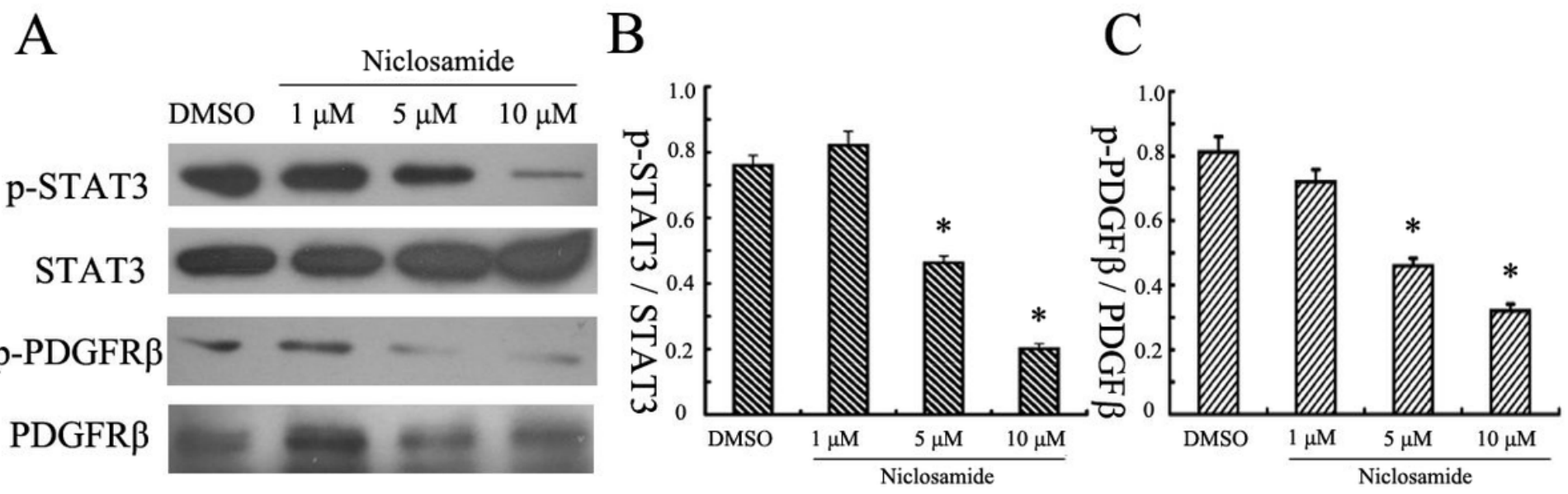

Figure 5

The expression of p-PDGFR $\beta$ and p-STAT3 was inhibited in SW1353 cells after being treated with the indicated concentrations of Niclosamide for $12 \mathrm{~h}$. Densitometric assays were presented as mean \pm standard as shown in the histogram. ${ }^{*} \mathrm{P}<0.05$ compared with control. 

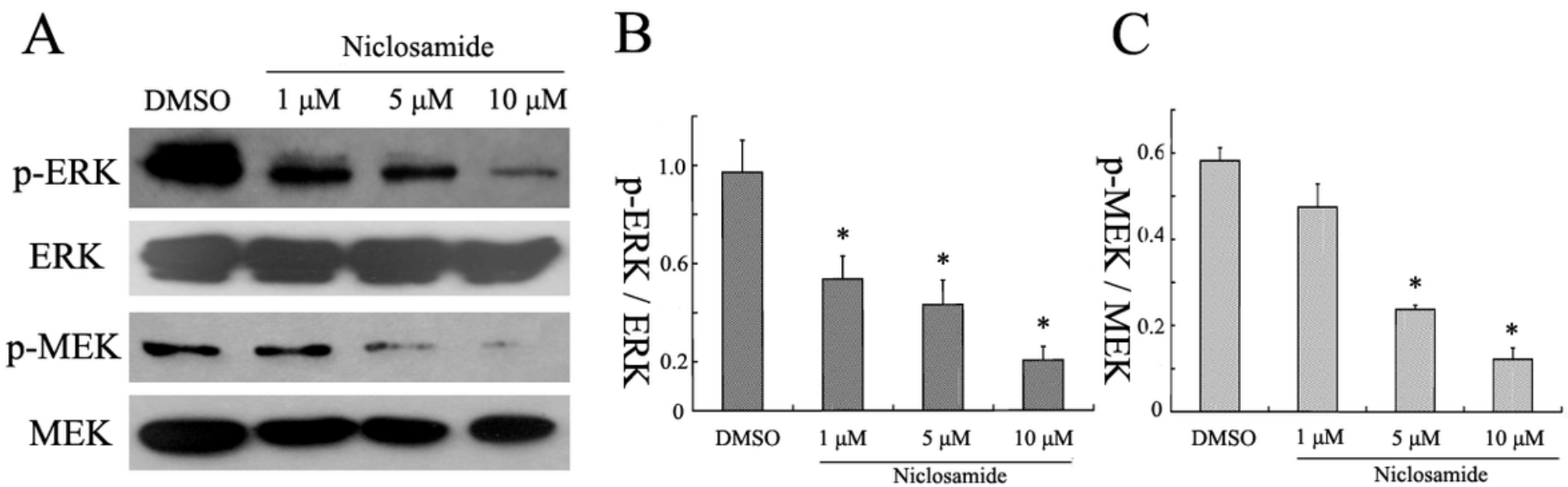

Figure 6

Niclosamide inhibits RAF/MEK/ERK signaling in SW1353cells. Cells were treated with the indicated concentrations of Niclosamide for $12 \mathrm{~h}$. Densitometric assays were performed and data were presented as mean \pm standard as shown in the histogram. ${ }^{*} \mathrm{P}<0.05$ compared with control.
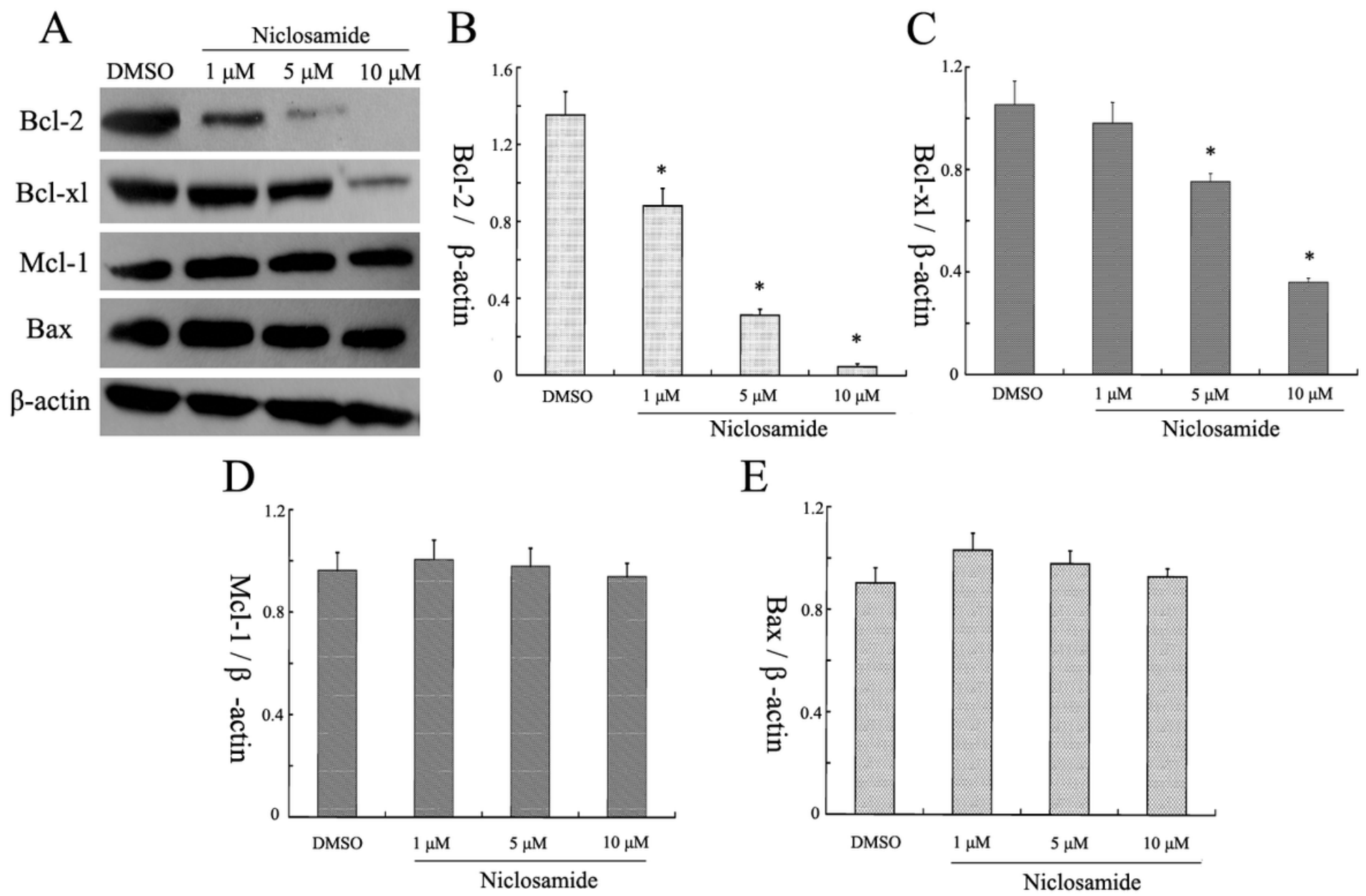

$\mathrm{E}$

Figure 7 
Niclosamide down-regulates Bcl-2 and Mcl-1 levels in SW1353 cells, but have little effect on the expression of Mcl-1 or Bax. $\beta$-actin was used as an internal control. Densitometric assays of proteins were performed and data were presented as mean \pm standard as shown in the histogram. ${ }^{*} P<0.05$ compared with control. 